\title{
GREENHOUSE GAS LASER IMAGING TOMOGRAPHY EXPERIMENT (GreenLITE)
}

\author{
Jeremy Dobler ${ }^{1 *}$, Nathan Blume ${ }^{1}$, Michael Braun ${ }^{1}$, T. Scott Zaccheo ${ }^{2}$, Tim Pernini ${ }^{2}$, and Chris \\ Botos $^{2}$
}

\author{
${ }^{1}$ Exelis Inc., Fort Wayne, IN 46818, USA, *Email: jeremy.dobler@exelisinc.com
}

${ }^{2}$ Atmospheric and Environmental Research Inc., Lexington, MA, USA

\begin{abstract}
Exelis has recently developed a novel laser-based instrument to aid in the autonomous real-time monitoring and mapping of $\mathrm{CO}_{2}$ concentration over a two-dimensional area. The Greenhouse gas Laser Imaging Tomography Experiment (GreenLITE) instrument uses two transceivers and a series of retroreflectors to continuously measure the differential transmission over a number of overlapping lines of sight or "chords", forming a plane. By inverting the differential transmission measurements along with locally measured temperature $(\mathrm{T})$, pressure $(\mathrm{P})$ and relative humidity $(\mathrm{RH})$ the average concentration of $\mathrm{CO}_{2}$ along each chord can be determined and, based on the overlap between chords, a 2D map of $\mathrm{CO}_{2}$ concentration over the measurement plane can be estimated. The GreenLITE system was deployed to the Zero Emissions Research and Technology (ZERT) center in Bozeman, Montana, in Aug-Sept 2014, where more than 200 hours of data were collected over a wide range of environmental conditions, while utilizing a controlled release of $\mathrm{CO}_{2}$ into a segmented underground pipe [1]. The system demonstrated the ability to identify persistent $\mathrm{CO}_{2}$ sources at the test facility and showed strong correlation with an independent measurement using a LI-COR based system. Here we describe the measurement approach, instrument design, and results from the deployment to the ZERT site.
\end{abstract}

\section{INTRODUCTION}

Under a cooperative agreement with the National Energy Technology Laboratory (NETL), Exelis and our partners at Atmospheric and Environmental Research (AER) have been developing a system with the focus of supporting the identification of possible leak sources for pilot Ground Carbon Sequestration (GCS) sites. The resulting GreenLITE system provides continuous, quantified, 2D $\mathrm{CO}_{2}$ concentrations and flux over a large area with high accuracy. It is based on proven technology utilized in a new and innovative way. The system uses two laser sensors, located 2-3 m off the ground, nominally preprogrammed to point to a series of 20 retroreflector targets each, as notionally depicted in Figure 1. This sensor target system provides an interwoven series of high accuracy, integrated path differential absorption measurements that are subsequently ingested into a tomographic retrieval algorithm used to generate a 2D reconstruction of the $\mathrm{CO}_{2}$ concentration across an entire carbon storage field. In addition the system was designed to operate remotely with minimal interaction, and all of the data processing and display is handled through a custom open web interface.

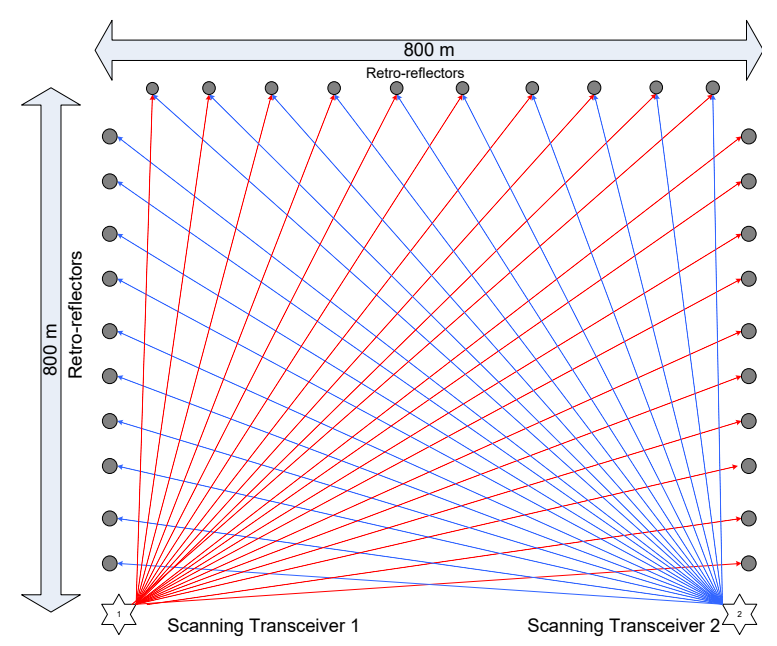

Figure 1: Notional GreenLITE configuration with 2 transceivers, 30 retroreflectors, and 20 chords per transceiver.

The laser sensor technology is based on more than a decade's worth of investments and experience developing and testing the Exelis Multifunctional Fiber-Laser Lidar (MFLL), an airborne instrument that has demonstrated measurements of column-integrated $\mathrm{CO}_{2}$ from 3 different aircraft over a wide range of conditions. The Exelis-built system has been under evaluation by NASA Langley Research Center since 2005 , and recent flights $(2010,2011,2013$ \& 2014) have demonstrated accuracies for the remote measurement of better than $0.67 \mathrm{ppmv}$, on average, with a precision of $1.7 \mathrm{ppmv}$ when compared to an onboard in situ monitor traceable to World Meteorological Organization (WMO) standards [2,3].

In addition to MFLL, Exelis has developed a novel bistatic lidar approach which has ground-to-ground and geostationary orbit-to-ground capability. This system is referred to as the Laser Atmospheric Transmitter Receiver-Network (LAnTeRN), and a proof of concept 
was recently (2012) demonstrated through Exelis internal research and development funding which supported the modeled performance estimates at extremely low signal levels [4]. Both systems, MFLL and LAnTeRN, rely on a unique single beam amplitude modulation approach and a digital lock-in amplifier to separate and measure the small signals out of a large background. The system being developed in this work takes advantage of the best features of both instruments.

\section{METHODOLOGY}

The measurement concept is based on Integrated Path Differential Absorption (IPDA) Laser Absorption Spectroscopy (LAS) [5,6]. In this approach two or more narrow-line $(\sim 4 \mathrm{MHz})$ laser wavelengths are positioned on a vibrational-rotational absorption feature of the desired molecule, such that at least one of the lines is in a highly absorbed region and at least one line is in a region of little or no absorption due to molecular vibration/rotation. The wavelengths are spaced very closely $(50-100 \mathrm{pm})$ and thus experience nearly identical extinction as they traverse an atmospheric path, except for the extinction due to absorption by the molecule of interest.

Using the Beer-Lambert law, if we know the transmitted power and the received power for a given wavelength we can get the Optical Depth $(\tau)$ through the relation $\mathrm{I} / \mathrm{I}_{0}=\exp (-2 \tau)$, where $\mathrm{I}$ is the received power, $I_{0}$ is the transmitted power, $\tau$ is the one way optical depth over the given path due to scattering and absorption, and the 2 accounts for the round trip in this configuration. By taking the ratio of the optical depth at the two closely spaced wavelengths, all terms in the $\tau$ fall out, or become negligible, other than the absorption difference $(\mathrm{d} \tau)$.

A unique and enabling aspect of the Exelis implementation is the simultaneous transmission and reception of multiple laser wavelengths using a Continuous Wave (CW) Intensity Modulated (IM) approach. In this approach each laser has a unique IM applied, and they are then combined in optical fiber before being transmitted to the atmosphere and subsequently received. The individual wavelength components are then separated after digitization using a custom digital lock-in amplifier or matched filter approach. This simultaneity causes several noise sources due to the atmosphere and instrument to become common-mode and fall out in the ratio, unlike other common pulsed or frequency swept approaches.

If the cross-section of the molecule is known, the difference in absorption over the path between the two wavelengths is proportional to the total number of molecules along the path, or chord. Additionally if the surface pressure and the path length are known, the average concentration of the molecule in the path can be determined based on an iterative approach that compares the differences between the observed values of $\tau$ with those computed via the Line-by-Line Radiative Transfer Model (LBLRTM) [7] developed by AER. The IPDA method results in an integrated column molecular number density value between the transmitter and the receiver $[5,6]$.

For the GreenLITE concept a large number of these density values are obtained over multiple intersecting chords, defined by the line between a transceiver and a retroreflector, as illustrated in Figure 1. An estimate of the $2 \mathrm{D}$ spatial concentration of $\mathrm{CO}_{2}$ within the field of regard can be determined using the grid of average concentration per chord and an analytic model that describes the underlying field distribution [8]. This is essentially a sparsely sampled tomographic problem. Our current approach assumes a limited number of Gaussian plumes, which can have different amplitudes and be stretched or rotated along a given axis. The solution is then iterated using a maximum descent approach to arrive at the solution that results in the smallest residual error between the observed differential transmission and retrieved concentrations.

\section{SYSTEM DESCRIPTION}

The transceivers consist of two main sections: a "hotbox" and the optics head. The hotbox houses the electronics, lasers, modulators, computers and communications equipment; is water tight; and maintains everything at an ambient temperature of 38 ${ }^{\circ} \mathrm{C}$. The optics head, which houses the transmit/receive optics and a camera, is also sealed and heated.

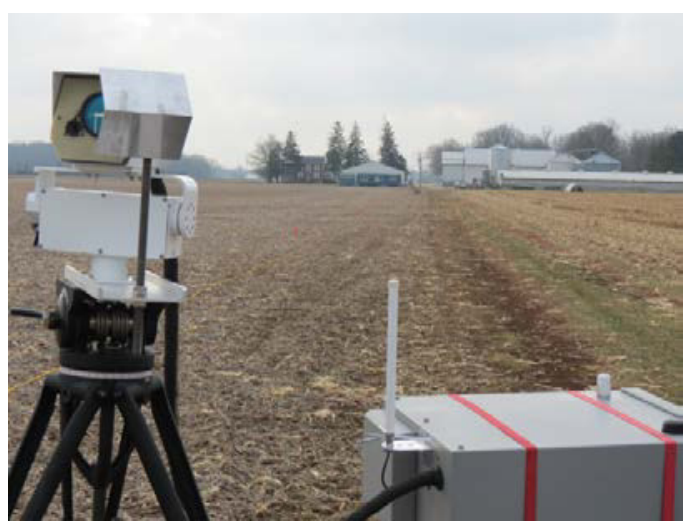

Figure 2: GreenLITE transceiver hardware at Exelis Farm test site in New Haven, IN.

The optics head is attached to a mechanical scanner which points the transceiver at the specified retroreflector based on a map programmed at setup. The scanner is capable of being reprogrammed 
remotely. The optics head and the hotbox are connected by a watertight hose containing the electrical cables and optical fibers. An additional component is the calibration target which allows the system to take a zero path measurement, where the ratio of the channels should be one, in order to allow monitoring of the instrument stability over time.

The system is designed for continuous remote operation. A list of the key system parameters is included in Table 1.

Table 1: Key system parameters of GreenLITE system.

\begin{tabular}{|l|l|}
\hline Online Wavelength & $1571.1129 \mathrm{~nm}$ \\
\hline Offline Wavelength & $1571.0629 \mathrm{~nm}$ \\
\hline Optical Transmit Power & $5 \mathrm{~mW}(2.5 \mathrm{~mW}$ per ch.) \\
\hline Modulation Rate & $19.2-24.6 \mathrm{kHz}$ \\
\hline Modulation Waveform & sinusoidal \\
\hline Sampling Rate & $1.0 \mathrm{MHz}$ \\
\hline Sampling Resolution & $16 \mathrm{bits}$ \\
\hline Transmitter Optics & $25 \mathrm{~mm}$ \\
\hline Receiver Optics & $25 \mathrm{~mm}$ \\
\hline Optics Configuration & biaxial, fiber-coupled \\
\hline Detector & InGaAs PIN \\
\hline TIA Gain & $10^{5}$ or $10^{6}$ (programmed) \\
\hline Retroreflectors & $50 \mathrm{~mm}$ \\
\hline Lockin Period & $10 \mathrm{sec}$ (adjustable) \\
\hline Power & $110 \mathrm{~V}, 60 \mathrm{~Hz}, 3 \mathrm{~A}$ \\
\hline
\end{tabular}

An additional key component of GreenLITE, in terms of performance enhancements, is the real time webbased processing and user interface. These web-based tools allow remote autonomous deployment of the system and handle all data storage, processing and dissemination of the data to the user. The user can explore the 2D reconstruction and individual chord data in real time, or scroll through a select period of time. Additionally, users can get information on the raw data and the instrument performance and health,

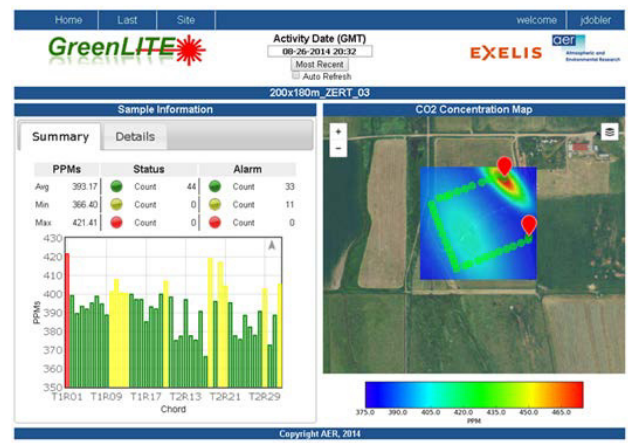

Figure 3: Example of GreenLITE web-based interface showing live individual chord concentration on left and the associated $2 \mathrm{D}$ reconstruction with geolocation on the right. either in real time or at selected times in the past.

\section{RESULTS}

The GreenLITE system provides a direct measurement of the $\mathrm{CO}_{2}$ spatial distribution across a large area, which can be used to identify and locate areas of persistent higher concentrations indicative of a source, such as a leak from a GCS site. This was seen in the ZERT testing of the GreenLITE system where higher concentration areas were repeatedly identified over an underground pipe used for the controlled release and near a large manure pile just off the edge of the site. The manure pile was measured independently using a LI-COR based system and was found to be producing spikes that were 10X larger than the concentration spikes being generated by the controlled release of $0.3 \mathrm{~T} /$ day at the height above ground used for the GreenLITE measurements ( $\sim 6 \mathrm{ft})$.

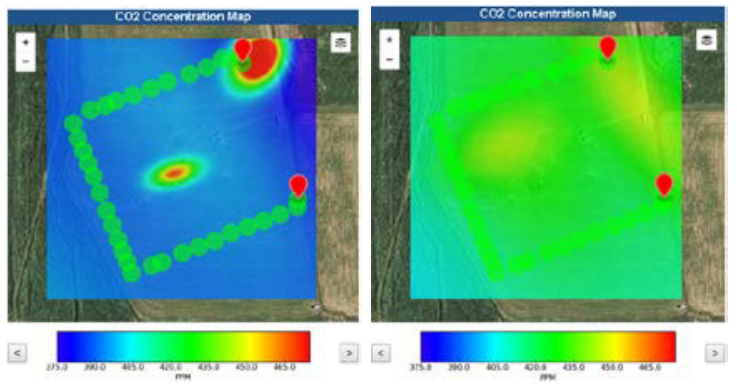

Figure 4: Measurements at the ZERT site on 27 Aug 2014, showing signature from ground release and from an adjacent manure pile (upper right corner of images). Scale goes from $375 \mathrm{ppm}$ to $475 \mathrm{ppm}$. Left image was taken in the afternoon and right image was taken at 7 am showing the difference in background due to vegetation drawdown and respiration, respectively.

The LI-COR system was a West system designed for chamber measurements. During the ZERT testing, the

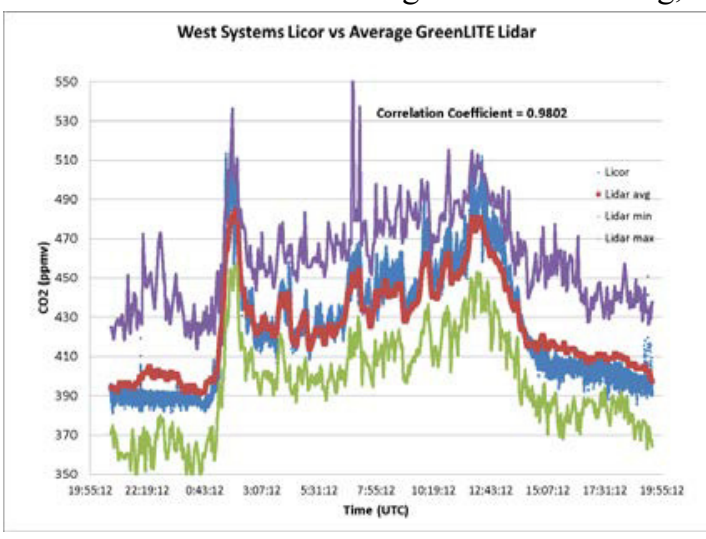

Figure 5: Comparison of average of all GreenLITE chords (red) to an independent LI-COR based measurement system (blue) over a 23 hour period with max and min chord values in purple and green, respectively. 
chamber was placed on a rebar post away from the underground pipe, in order to provide an independent measurement of the variability of the background $\mathrm{CO}_{2}$. Figure 5 shows the comparison of the LI-COR data to the lidar data. The correlation coefficient over this 23 hour period was 0.98 . Several other comparisons were made and showed correlations of $>0.94$ with the lidar data.

Evaluation of the instrument ratio using $10 \mathrm{~s}$ averages showed SNR values typically $>3000$ in the differential absorption ratio. Measured values over a $1400 \mathrm{~m}$ path were $>4000$ which is equivalent to $<1 \mathrm{ppm}$ precision. Realized setups at the ZERT site and local testing at the Exelis Farm test site required the use of path lengths much shorter than the design range of $1.2 \mathrm{~km}$, resulting in low absorption over the path and precisions ranging from 2-10 ppm per $10 \mathrm{~s}$ measurement.

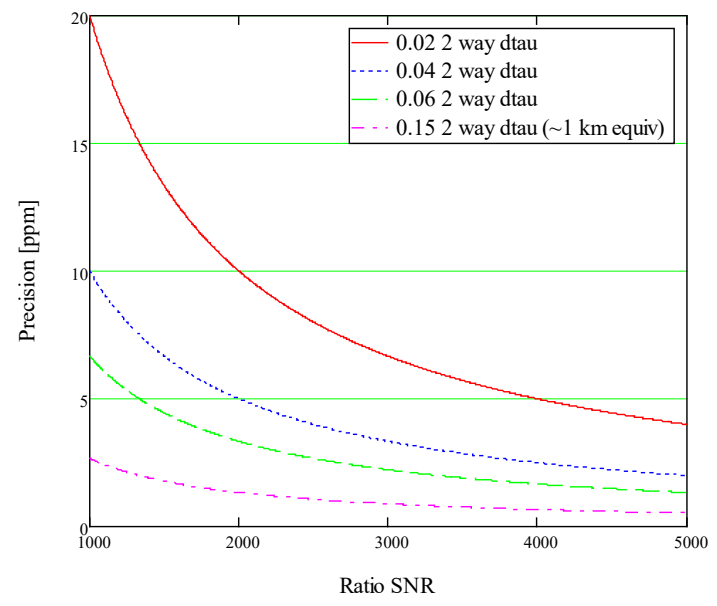

Figure 6: Trade between low differential optical depth $(\mathrm{d} \tau)$ for short paths and SNR versus precision

\section{CONCLUSIONS}

The GreenLITE system has demonstrated the ability to generate $2 \mathrm{D}$ spatial concentration maps of $\mathrm{CO}_{2}$ to aid in the identification and location of likely sources. The system was tested at the Exelis Farm test site in New Haven, IN, and is exceeding the design requirements. It has also been tested using a quantifiable underground leak source at the ZERT site in Bozeman, MT, and was able to identify persistent elevated $\mathrm{CO}_{2}$ sources even in the presence of large background fluctuations. To further test the system's utility and robustness, it has recently been deployed to an industrial GCS site in Illinois, where it is currently running autonomously, uploading measurement data to the web-based processing, and displaying system data and 2D reconstructions in near real-time.

Exelis and AER are in the process of scaling the measurement capability to $5 \mathrm{~km}$ scales to enhance the applicability to a wider range of GCS sites, and are evaluating the technology for other applications such as urban areas, or facilities monitoring.

\section{ACKNOWLEDGEMENT}

Funding for this work has been partially provided by the National Energy Technology Laboratory under the Department of Energy cooperative agreement \# DEFE0012574. We would also like to acknowledge Dr. Zachary Levine for assistance and discussion regarding more advanced approaches for estimating the 2D reconstructions.

\section{REFERENCES}

[1] Spangler, L. E., et al, 2009: A shallow subsurface controlled release facility in Bozeman, Montana, USA, for testing near surface $\mathrm{CO} 2$ detection techniques and transport models, Environmental Earth Sciences, 60 (2), 227-239.

[2] Browell, E. V., Dobler, J., Kooi, S., Choi, Y., Harrison, F. W., Moore, B., \& Zaccheo, T. S., 2010: Airborne validation of laser remote measurements of atmospheric carbon dioxide, 25th International Laser Radar Conference, St. Petersburg, Russia, July 5-9.

[3] Dobler, J. T., Harrison, F. W.; Browell, E. V.; Lin, B.; McGregor, D. P.; Kooi, S. A.; Choi, Y.; Ismail, S., 2013: Atmospheric CO2 column measurements with an airborne intensity-modulated continuous wave $1.57 \mu \mathrm{m}$ fiber laser lidar, Appl. Opt., 52, 2874-2892.

[4] Dobler, J., Braun, M., Blume, N., Zaccheo, T. S., 2013: A new laser based approach for measuring atmospheric greenhouse gases, Remote Sens., 5, 62846304.

[5] Korb, C. L., Weng, C. Y., 1983: Differential absorption lidar technique for measurement of the atmospheric profile, Applied Optics, 22(23), 37593770 .

[6] Measures, R., 1992: Laser remote sensing: fundamentals and applications, Krieger Publishing Company, New York, 243-247 and 305-310.

[7] Clough, S. A.; Shephard, M. W.; Mlawer, E. J.; Delamere, J. S.; Iacono, M. J.; Cady-Pereira, K.; Boukabara, S.; Brown, P. D., 2005: Atmospheric radiative transfer modeling: a summary of the AER codes, Short Communication. J. Quant. Spectrosc. Radiat. Transfer, 91, 233-244.

[8] Giuli, D., Toccafondi, A., Biffi Gentili, G., Freni, A., 1991: Tomographic reconstruction of rainfall fields through microwave attenuation measurements, J. Appl. Meteorol., 30, 1323-1340. 